\title{
THREE-DIMENSIONAL NEURITE TRACING UNDER GLOBALLY VARYING CONTRAST
}

\author{
S. Gulyanon ${ }^{1}$, N. Sharifai ${ }^{2,3}$, S. Bleykhman ${ }^{4}$, E. Kelly ${ }^{5}$, M.D. Kim ${ }^{6}$, A. Chiba ${ }^{3}$, and G. Tsechpenakis ${ }^{1}$ \\ ${ }^{1}$ Computer and Information Science Department, Indiana University-Purdue University Indianapolis, USA; \\ ${ }^{2}$ University of Miami Miller School of Medicine, USA; ${ }^{3}$ Biology Department, University of Miami, USA; \\ ${ }^{4}$ Carmel High School, IN, USA; ${ }^{5}$ Brebeuf Jesuit Preparatory School, IN, USA; \\ ${ }^{6}$ Molecular and Cellular Pharmacology Department, University of Miami, USA
}

\begin{abstract}
We study the 3D neurite tracing problem in different imaging modalities. We consider that the examined images do not provide sufficient contrast between neurite and background, and the signal-to-noise ratio varies spatially. We first split the stack into box sub-volumes, and inside each box we evolve simultaneously a number of different open-curve snakes. The curves deform based on three criteria: local image statistics, local shape smoothness, and a term that enforces pairwise attraction between snakes, given their spatial proximity and shapes. We validate our method using larva Drosophila sensory neurons imaged with confocal laser scanning microscopy, as well as publicly available datasets.
\end{abstract}

Index Terms - neurite tracing, neuron morphology, Drosophila, snakes

\section{INTRODUCTION}

Neuronal morphology is important for studying the structure and function of neurons, as well as their connectivity. The first steps towards encoding such information into a compact numerical representation is locating the neuron in images, identifying its parts (soma, axon, dendrites), and tracing axon and dendrite branches (what we summarize with the term neurite) [8]. The latter task has been a major challenge mainly due to imaging device limitations, image noise, as well as structural complexity and variability of the neurites.

There is a large number of neurite reconstruction approaches, many of which have been reviewed in [8] and [11]. They can be categorized into global, local, and combinations of the two. Global methods usually extract skeletons of neurites based on global intensity distribution of the images. Such methods involve, in general, measuring vesselness, e.g., [5], binarization, and/or skeletonization, as in [12]. Local methods extract the neurite centerline by starting from seeds, which are located automatically or manually, and iteratively perform tracing based on intensity statistics $[10,9,11]$. Although most of these methods have been shown to perform

This work is supported by NSF/DBI [\#1252597]: 'CAREER: Modeling the structure and dynamics of neuronal circuits in the Drosophila larvae using image analytics' awarded to G. Tsechpenakis.
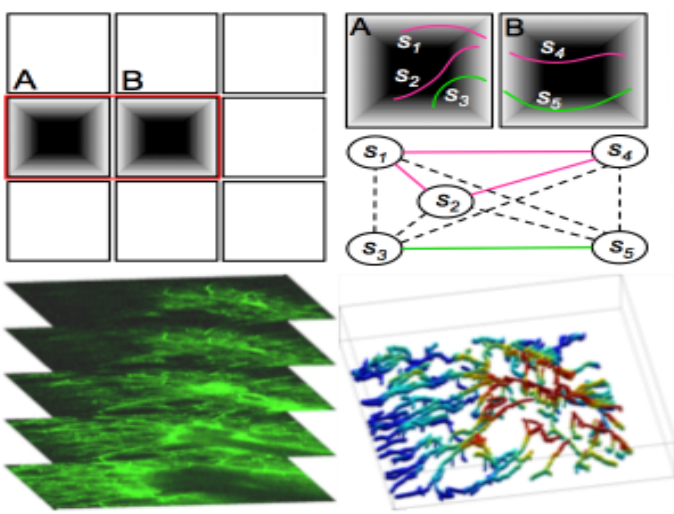

Fig. 1: Our approach. A stack is divided into box sub-volumes (top left). In each box, we evolve open-curve snakes based on local image features, shape smoothness, and snake interactions, regulated by proximity and shapes (top right): $\left(s_{1}, s_{2}, s_{4}\right)$ are close to each other and have compatible shapes; $\left(s_{3}, s_{5}\right)$ do not appear to belong to the same branch, and therefore do not interact with $\left(s_{1}, s_{2}, s_{4}\right)$. Under noise, we can successfully reconstruct neurites in $3 \mathrm{D}$ (bottom).

sufficiently on specific data, there is one common drawback that limits their generalization: they usually perform poorly, if the examined image stack depicts spatially varying signalto-noise ratios and contrast (due to, e.g., imaging modality limitations, presence of surrounding structures, etc.). In this work we aim at tackling this issue with a hybrid model-based framework.

Specifically, we split the image stack into box subvolumes, where the neurite/background intensity can be sufficiently classified. Within each sub-volume we start from initial seeds, detected by classification, and we evolve different open-curve snakes based on three criteria: (i) local intensity, (ii) individual curve shape smoothness, and (iii) interactions between neighboring snakes, based on their proximity and local shapes, including snakes in neighboring sub-volumes (Fig. 1). We formulate the solution as a maximum a posteriori probability estimation, where the objective is a joint probability of the snake population configuration (positions and shapes) and the labels of the voxels (neurite vs background). We validate our method using two different types of data: sensory neurons from larva Drosophila, and different

This is the author's manuscript of the article published in final edited form as:

Gulyanon, S., Sharifai, N., Bleykhman, S., Kelly, E., Kim, M. D., Chiba, A., \& Tsechpenakis, G. (2015). Three-dimensional neurite tracing under globally varying contrast. In 2015 IEEE 12th International Symposium on Biomedical Imaging (ISBI) (pp. 875-879).

http://doi.org/10.1109/ISBI.2015.7164010 
datasets from the DIADEM challenge ${ }^{1}$ [2]. Here we compare our method with three of the DIADEM challenge finalists, namely the neural circuit tracer [4], the principle curve tracing [1], and the 3D snake model in [11].

\section{METHOD}

Let the data volume $\mathbf{V} \in \mathcal{R}^{3}$, partitioned into non-overlapping box sub-volumes. Also let $V^{i}$ and $V^{j}, i \neq j$, denote two adjacent boxes, considering locally 27-box neighborhoods. Within each box, we detect seed points probabilistically, as we describe below; these seeds are the initialization for a set of snakes that we evolve locally.

Let $S^{i}$ be the set of snakes in sub-volume $V^{i}, S^{i}=$ $\left\{s_{n}\right\}_{n=1}^{N}$, and $\mathbf{S}=\bigcup_{k=1}^{K} s_{k}$ denote the set of all snakes in the box-neighborhood $(K \geq N)$. There can be attraction between any two curves in $S^{i} \bigcup S^{j}, i \neq j$, given (a) that $V^{i}$ and $V^{j}$ are adjacent sub-volumes, (b) their close spatial proximity and their compatible shape configurations (Fig. 1).

On the other hand, every voxel $v \in \mathbf{V}$ can be classified as neurite or background, i.e., $y(v)=\{+1,-1\}$, where $y$ is the binary variable of the voxel label. Let $Y^{i}=\{y(v)\}_{v \in V^{i}}$ be the set of voxel labels in sub-volume $V^{i}$, and $\mathbf{Y}=\bigcup_{i=1}^{27} Y^{i}$ be all the labels in the box-neighborhood. Each $Y^{i}$ is calculated independently from the neighboring sub-volumes, to account for local feature variations.

Given the above notations, we formulate our objective locally as a joint optimization of the snake configuration and voxel labels,

$$
\langle\hat{\mathbf{S}}, \hat{\mathbf{Y}}\rangle=\arg \max _{\langle\mathbf{S}, \mathbf{Y}\rangle} P(\mathbf{S}, \mathbf{Y} \mid \mathbf{X}),
$$

where $\mathbf{X}=\bigcup_{i=1}^{27} X^{i}$ denotes the features calculated from the data, and the objective joint conditional probability is,

$$
\begin{aligned}
P(\mathbf{S}, \mathbf{Y} \mid \mathbf{X}) & \propto P(\mathbf{X} \mid \mathbf{Y}) \cdot P(\mathbf{Y} \mid \mathbf{S}) \cdot P(\mathbf{S}) \\
& \propto P(\mathbf{X}) \cdot P(\mathbf{Y} \mid \mathbf{X}) \cdot P(\mathbf{Y} \mid \mathbf{S}) \cdot P(\mathbf{S})
\end{aligned}
$$

The data prior $P(\mathbf{X})$ is expressed locally as a Gaussian distribution. Here, as data features we use indicatively the Frangi filter responses [5], however, one may use other vesselness/tubularity features. Thus, we define,

$$
P\left(x^{i}(v)\right)=\frac{1}{\sqrt{2 \pi \sigma_{0}^{2}}} e^{-\left[x^{i}(v)\right]^{2} / \sigma_{0}^{2}},
$$

where $x^{i}(v)$ is the Frangi filter response at the location $v \in$ $V^{i}$. Again, to calculate such features, we consider each subvolume independently, as separate data.

The factor $P(\mathbf{Y} \mid \mathbf{X})$ is essentially the data term that drives the snake evolution in the vicinity. It is calculated independently within each box sub-volume, as $P\left(Y^{i} \mid X^{i}\right)$, and therefore there is no probability smoothness imposed between neighboring boxes, with respect to spatial distribution. The reason for such choice is to consider each box as separate data (stack), and calculate probabilities based only on local

\footnotetext{
${ }^{1}$ Datasets and metrics are available at: diademchallenge.org
}

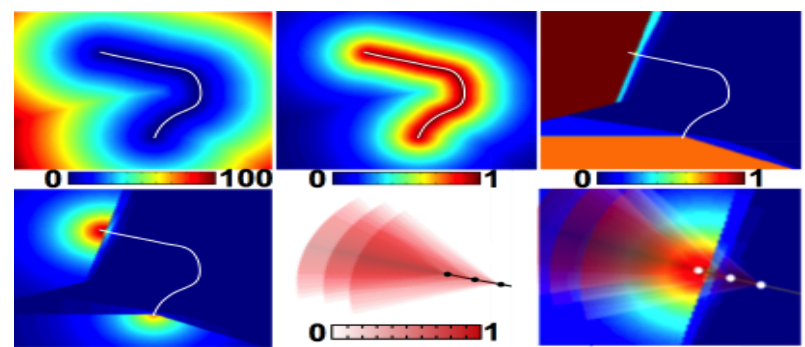

Fig. 2: Illustration of how a single snake participates in the attraction energy. Top (from left to right): distance map $d_{n}, D_{n}$ in eq. (8), and $F_{n}$ in eq. (10). Bottom (from left to right): $D_{n} F_{n}, G_{n}$ in eq. (12), and $D_{n} F_{n} G_{n}$. In eq. (13) we use $\left(1-D_{n}\right)\left(1-F_{n}\right)\left(1-G_{n}\right)$ for energy minimization.

intensity (Frangi response) statistics. To calculate such probability fields, we use a Discriminative Random Field (DRF) [7], where we solve inference with the Highest Confidence First algorithm [3], due to its approximation accuracy and efficiency. In our implementation, we used the positively classified voxels with high confidence as initial seeds that determine the snake population.

The term $P(\mathbf{Y} \mid \mathbf{S})$ encodes the likelihood of voxels being part of the neurite, given the configuration of the snakes. If $d_{k}$ is the (unsigned) distance function for each snake $s_{k} \in \mathbf{S}$,

$$
\begin{gathered}
P\left(y(v) \mid s_{k}\right)=e^{-d_{k}(v)}, \text { and } \\
P(y(v) \mid \mathbf{S})=\max \left\{P\left(y(v) \mid s_{1}\right), \ldots, P\left(y(v) \mid s_{K}\right)\right\}
\end{gathered}
$$

Finally, $P(\mathbf{S})$ corresponds to the local smoothness of the models and their pairwise attractions,

$$
P\left(S^{i}\right)=e^{-E_{s}\left(S^{i}\right)-E_{a}\left(S^{i}\right)},
$$

The smoothness energy for the population $S^{i}$ in $V^{i}$ is, $E_{s}\left(S^{i}\right)=$

$$
\sum_{n=1}^{N}\left\{\int_{0}^{1}\left[\alpha(q)\left\|\nabla s_{n}\right\|^{2}+\beta(q)\left\|\nabla^{2} s_{n}\right\|^{2}\right] d q\right\},
$$

with $q$ encoding the normalized arc-length of each snake, and $(\alpha, \beta)$ being the elasticity and rigidity weights; we consider $\alpha=\beta=1$ for all snakes in the entire volume.

The effect of attraction energy $E_{a}$ in eq. (6) is shown in Fig. 1. Each point at a (normalized) location $q$ along a snake, contributes to an attraction field, i.e., an energy that drives the extension of all snakes towards certain directions.

Let us consider a single model $s_{n} \in S^{i}$ and how it affects its surroundings. Using its distance function $d_{n}$, we define,

$$
D_{n}(v)=e^{-\gamma d_{n}(v)}, 0 \leq \gamma \leq 1,
$$

which implies that the further a location $u$ from $s_{n}$, the lower the degree of influence at $u$ from that model. We use $\gamma$ to control the slope of the exponential: higher $\gamma$ values produce narrower zones of influence around the curve (Fig. 2).

We also consider that only the end-parts of the snake can create attraction; therefore, we define the functional that assigns weights to curve points as,

$$
f(q)=e^{-\delta q}+e^{-\delta(1-q)}, q \in[0,1],
$$




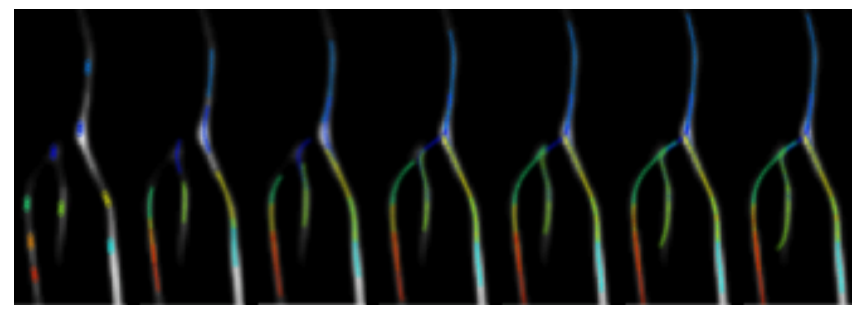

Fig. 3: Evolution instances of different snakes (in colors). From left to right: iteration $\sharp 5,10,15,20,25,30$ and 40 (termination). The images have been enhanced for illustration.

which imposes higher attraction by points at the two curve ends, and practically no influence by intermediate points. We calculate the map (Fig. 2),

$$
F_{n}(v)=\left\{f\left(q_{0}\right)|| v\left(q_{0}\right)-v \mid=d_{n}(v)\right\},
$$

where $q_{0}$ is the location along the normalized arc length of the curve, with cartesian coordinates $v\left(q_{0}\right)$, closest to the voxel $v$.

We also determine an angle of influence $\theta$, with respect to the direction of the curve's first derivative. Every location $v$ can be affected by any point $q$ on the snake based on,

$$
\begin{aligned}
& g_{n}(v, q)= \\
& \left\{\begin{array}{ll}
\left|\cos \left(\left.\angle \nabla s_{n}\right|_{q}\right)-\cos \left(\left.\angle \nabla s_{n}\right|_{q}+\theta\right)\right|, & \theta \in[-\vartheta,+\vartheta] \\
0, & \text { otherwise }
\end{array},\right.
\end{aligned}
$$

where $\left.\angle \nabla s_{n}\right|_{q}$ is the tangential orientation at point $q$ on the curve, and $\theta$ is the angle between $\overline{v(q) v}$ and $\overline{\left.\nabla s_{n}\right|_{q}}$. In all our experiments we considered $\vartheta=\pi / 3$. We define (Fig. 2),

$$
G_{n}(v)=\prod_{q \in[0,1]} g_{n}(v, q),
$$

Using the definitions of eqs. (8), (10) and (12), since $0 \leq$ $D_{n}(u), F_{n}(v), G_{n}(v) \leq 1$, we formulate the attraction map of snake $s_{n}$,

$$
A_{n}(u)=\left(1-D_{n}(u)\right)\left(1-F_{n}(u)\right)\left(1-G_{n}(u)\right)
$$

where low values indicate high influence. Therefore, for any $s_{n} \in S^{i}$ and $s_{k} \in \mathbf{S}, s_{n} \neq s_{k}$,

$$
\mathcal{A}_{n k}(u)=\max \left\{A_{n}(u), A_{n}(u)\right\}, \forall u \in \bigcup_{i=1}^{27} V^{i}
$$

which essentially encodes that two models can be attracted to each other only when the highest value among the two attraction maps at location $u$ is low enough. The attraction energy of every curve $s_{n}$ in $V^{i}$ sources from all $\mathrm{K}$ snakes in the boxneighborhood as,

$$
e_{a}\left(s_{n}\right)=\int_{0}^{1}\left[\sum_{k=1}^{K} \mathcal{A}_{n k}(u(q))\right] d q, s_{k} \neq s_{n}
$$

and for the population $S^{i}=\left\{s_{n}\right\}_{n=1}^{N}$,

$$
E_{a}\left(S^{i}\right)=\sum_{n=1}^{N} e_{a}\left(s_{n}\right)
$$

Notice that all snakes in the box sub-volume neighborhood participate in this energy of the set $S^{i}$, given their pairwise proximity imposed by the terms $D$ (eq. (8)) and $F$ (eq. (10)).

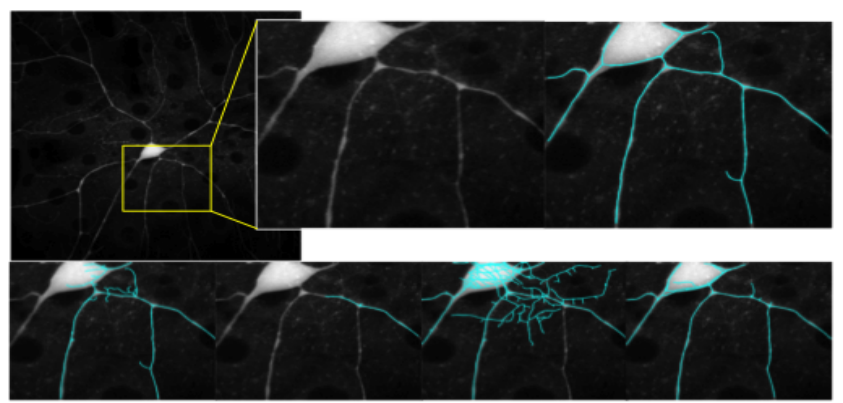

(a)

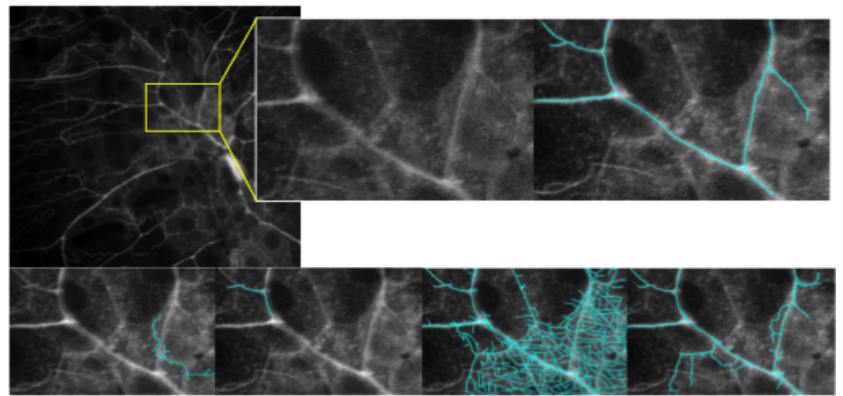

(b)

Fig. 4: Tracing of two Drosophila sensory neurons. Top rows in (a) and (b) (from left to right): collapsed stack image of the neuron, magnified region where the results are shown, and ground-truth (in cyan) from manual tracing. Bottom rows in (a) and (b), in cyan (left to right): NCT [4], PCT [1], 3D snake [11], and our method.

Using the definitions in eqs. (7), (16), we calculate the prior $P\left(S^{i}\right)$ in eq. (6). Then we solve the objective in eqs. (1), (2) iteratively, in a narrow band around each open curve. In eq. (2), the terms $P(\mathbf{Y} \mid \mathbf{X})$ and $P(\mathbf{Y} \mid \mathbf{S})$ are calculated within each box sub-volume separately, while $P(\mathbf{S})$ involves shape and location information from snakes across all neighboring sub-volumes. Fig. 3 illustrates in 2D the evolution of nine snakes, corresponding to the initial seeds detected by the DRF-based classification. For simplicity we consider two adjacent box-volumes with virtually no background noise.

\section{EXPERIMENTS}

We applied our method in data from two different sources: (a) 11 image stacks of sensory neurons in the wild-type larva Drosophila, for the study of dendritic arborization patterns over the four instars of development; (b) 13 stacks from three datasets in the DIADEM challenge. In both cases, we made comparisons with three DIADEM challenge finalists, namely the neuron circuit tracer (NCT) [4], the principal curve tracing (PCT) [1], and the 3D snake model in [11], using the metric in [6]. Due to space limitation we cannot include here numerical comparisons. However, we note that for all examined volumes, our approach gives better, and sometimes equally good, results with the considered competition.

\subsection{Sensory neurons in the larva Drosophila}

We prepared and imaged all the fruit fly samples. In our tracing software we integrated the calculation of dendritic arborization features, such as branching point locations, branch 

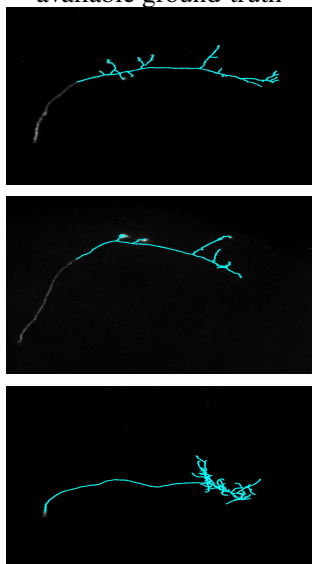

NCT [4]
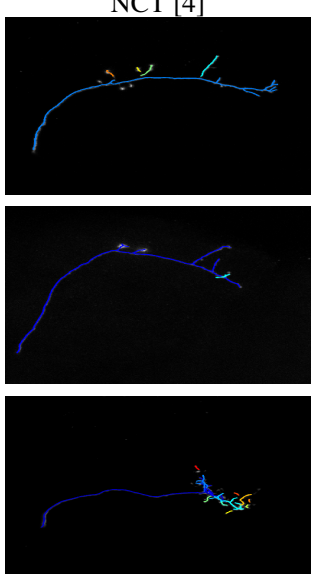

PCT [1]
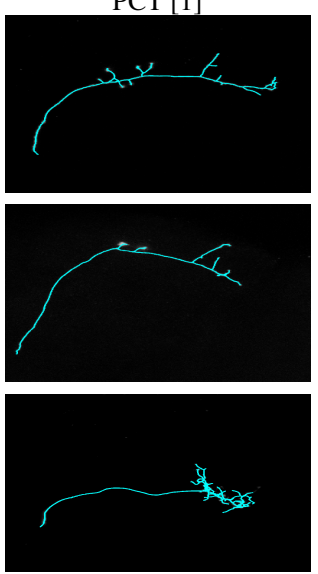

3D snake [11]
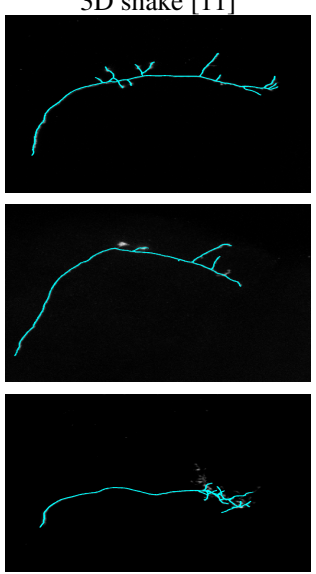

2.0our method
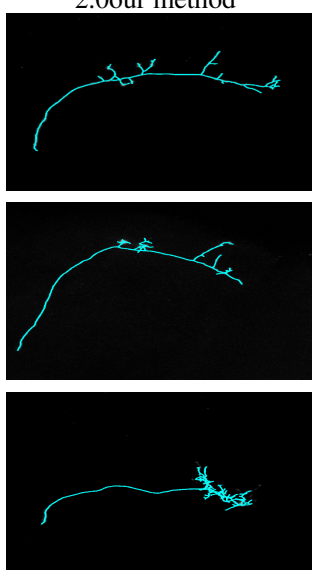

Fig. 5: Results (in color) on the DIADEM Olfactory Projection Fibers dataset. Rows correspond to different stacks (OP7, OP8, OP9). From left to right: ground truth, the results of NCT [4], PCT [1], 3D snake [11], and our method. Different colors show parts of different neurons.

lengths, principal directions of branches, etc., along with their coding as spatial distributions. We leave the dendritic features and their analysis for future discussion.

Fly propagation. Adult flies (ppk-GAL4 mCD8::GFP) were kept in vials and stored at room temperature. Vials contained fly feed composed of cornstarch, agar, molasses, and yeast.

Genetic crosses. 3-4 males and females were placed in mating cages to facilitate larval collection. Cages were loaded with grape agar plates containing two drops of live active yeast on the center of the agar plate. Agar plates were swapped at appropriate times to ensure correct age of larval development (72 hours for 1st day 3rd instar). Larvae were checked for typical morphological features to ensure correct age. The cages and plates were incubated at $25^{\circ} \mathrm{C}$.

Mounting. Larvae were mounted one per slide $(75 \times 25 \times$ $1 \mathrm{~mm}$ ) in Halocarbon $1000 \mathrm{~N}$ oil to match the refractive index of microscope objective oil-immersion fluid. Coverslips $(22 \times 22 \mathrm{~mm})$ were secured using putty in order to apply appropriate pressure without popping the larva and to prevent larva movement while imaging.

Anatomy. Larvae for all experiments were 48-72 hours old (2nd-3rd instar). The neurons used in imaging were on the distal left side along the larvae's dorsal end, within hemisegments $\mathrm{T} 2, \mathrm{~T} 3, \mathrm{~A} 1$, and $\mathrm{A} 2$.

Microscopy. Image stacks (.5 micron sections) of Class V da (dendritic arborization) sensory neurons were detected with an inverse confocal laser scanning microscope (Zeiss LSM 780) using a Plan-Neofluar $40 \times / 1.30$ Oil M27 objective (Zeiss) and the ZEN 2010 software. The system utilized an argon laser line (Ar-Laser Multiline 458/488/514 nm; Zeiss) for GFP excitation $(488 \mathrm{~nm})$ at $5 \%$ intensity, beam splitter MBS 488, and a pinhole size of 1 airy unit.

Tracing. In Fig. 4 we illustrate indicative tracing results of our approach, along with the three existing methods we used for comparisons. The two cases shown in (a) and (b) are typical examples of where our approach provides increased robustness, in the presence of varying contrast and noise.

\subsection{DIADEM challenge datasets}

We used 6 stacks from the Olfactory Projection Fibers set, 5 stacks from the Neocortical Layer 1 Axons set, and 2 stacks from the Cerebellar Climbing Fibers set [2], available online at the challenge's website.

Tracing. In these datasets it was not always obvious how much better our approach performed compared to the other three examined methods. In Fig. 5 we illustrate three cases where our method and the competition performed overall equally well. This is mainly due to the fact that in such data there is very little background variation, and we do not fully exploit our method's focus on local ambiguities.

\section{CONCLUSION}

In this work we examined the problem of 3D neurite tracing, under varying noise. Our work is based on open-curve snakes, that evolve locally, driven by local data features, shape smoothness, and pairwise attractions with nearby snakes. The user can introduce different data features, and can consider different resolutions of locality: we split the volume into box sub-volumes within which we solve the evolution of the snake population; different sizes of such subvolumes can be considered. We leave for future discussion result comparisons when using different sub-volume sizes and different data features. We compared our method with existing approaches, using larval Drosophila sensory neurons imaged with laser scanning confocal microscopy, and publicly available datasets. From the examined data, it appears that our method can be confidently used for different imaging modalities, for quantifying dendritic features, necessary for describing numerically neuronal morphology and its patterns.

\section{REFERENCES}

[1] E. Bas and D. Erdogmus, 'Principal curves as skeletons of tubular objects: locally characterizing the structures of axons,' $\mathrm{Neu}$ roinformatics, 9(2-3):181-191, 2011. 
[2] K.M. Brown, G.Barrionuevo, A.J. Canty, V. DePaola, J.A. Hirsch, G.S.X.E. Jefferis, J. Lu, M. Snippe, I. Sugihara, and G.A. Ascoli, 'The DIADEM data sets: representative light microscopy images of neuronal morphology to advance automation of digital reconstructions,' Neuroinformatics, 9(2-3):143157, 2011.

[3] P. Chou and C. Brown, 'The theory and practice of bayesian image labeling,' Intl J. of Computer Vision, 4:185-210, 1990.

[4] P. Chothani, V. Mehta, and A. Stepanyants, 'Automated tracing of neurites from light microscopy stacks of images,' Neuroinformatics, 9(2-3):263-278, 2011.

[5] A.F. Frangi, W.J. Niessen, K.L. Vincken, and M.A. Viergever, 'Multiscale vessel enhancement filtering,' MICCAI, pp. 130137, 1998.

[6] T.A. Gillette, K.M. Brown, and G.A. Ascoli, 'The diadem metric: comparing multiple reconstructions of the same neuron,' Neuroinformatics, 9(2-3):233-245, 2011.

[7] S. Kumar and M. Hebert, 'Discriminative Random Fields,' Int'l J. of Computer Vision, 68(2):179-201, 2006.

[8] E. Meijering, 'Neuron tracing in perspective,' Cytometry, 77A(7):693-704, 2010.

[9] J.A. Mitchel, I.S. Martin, and D. Hoffman-Kim, 'Neurient: An algorithm for automatic tracing of confluent neuronal images to determine alignment,' Neurosci. Meth., 214(2):210-222, 2013.

[10] E. Türetken, G. González, C. Blum, and P. Fua, 'Automated reconstruction of dendritic and axonal trees by global optimization with geometric priors,' Neuroinformatics, 9(2-3):279-302, 2011.

[11] Y. Wang, A. Narayanaswamy, C.-L. Tsai, and B. Roysam, 'A broadly applicable $3 \mathrm{~d}$ neuron tracing method based on opencurve snake,' Neuroinformatics, 9(2-3):193-217, 2011.

[12] T. Zhao, J. Xie, F. Amat, N.G. Clack, P. Ahammad, H. Peng, F. Long, and E. W. Myers, 'Automated reconstruction of neuronal morphology based on local geometrical and global structural models,' Neuroinformatics, 9(2-3):247-261, 2011. 